This is the accepted manuscript of the article, which has been published in Early

child development and care. Published online: 12 May 2019.

https://doi.org/10.1080/03004430.2019.1613651

\title{
Shared Pleasure in early mother-infant interactions: A study in a high-risk South African sample
}

\section{Author details}

Anusha Lachman, Department of Psychiatry, Stellenbosch University. Email: anusha@sun.ac.za, ORCID ID: https://orcid.org/0000-0002-7593-2916

Dana JH Niehaus, Department of Psychiatry, Stellenbosch University. Email: djhn@sun.ac.za, ORCID ID: https://orcid.org/0000-0001-9696-5605

Esme R Jordaan(), Biostatistics unit, South African Medical Research Council, Parow, South Africa and Statistics and Population Studies, University of the Western Cape. Email: Esme.Jordaan@mrc.ac.za ORCID ID: https://orcid.org/0000-0002-0361-3473

Jukka Leppanen, Child Psychiatry, Tampere University, Finland. Email: jukka.leppanen@uti.fi ORCID ID:

Kaija Puura, Child Psychiatry, Tampere University, Finland. Email: Kaija.Puura@pshp.fi ORCID ID: https://orcid.org/0000-0001-6707-7975

\section{Corresponding author}

Anusha Lachman

Department of Psychiatry, Tygerberg Campus, Stellenbosch University

Francie van Zyl Drive, Cape Town, 7550

anusha@sun.ac.za

\section{Word count 6646}




\title{
Shared Pleasure in early mother-infant interactions: A study in a high-risk South African sample
}

\begin{abstract}
Background: Infant mental health is strongly connected to an infant's relationship with a responsive, warm, and available caregiver. However, maternal mental illness reduces a mother's ability to detect and respond to changes in her infant's expressions and communication, which may have important consequences for infant attachment and emotion regulation. The Shared Pleasure (SP) paradigm in parent-infant interactions is defined as "the parent and the child sharing positive affect in synchrony” and is considered to be a possible screening marker for early identification of at-risk dyads. However, a paucity of data exists for the application of SP as a measurable paradigm in developing countries.
\end{abstract}

Aim: To evaluate the Shared Pleasure Paradigm using women attending a tertiary psychiatric maternal mental health clinic in Cape Town, South Africa.

Methods: A sample of mothers ( $\mathrm{N}=78)$ and young infants (2-6months old) attending a Maternal Mental Health Clinic were assessed for SP moments using video recordings of the dyad in free play.

Results: SP moments occurred in only $20.5 \%$ of the sample. SP moments were more frequent in younger babies (under 3 months of age). There were significantly more SP moments in dyads where mothers had no mental illnesses $(p=0.021)$ or were married $(p=0.016)$. Black African mothers also experienced significantly more SP moments with their babies $(p=0.033)$ than their Caucasian or Mixed-ancestry counterparts.

Conclusions: This study explored the application of the "Shared Pleasure Paradigm" on women with and without mental illness who attended a tertiary psychiatric maternal mental health clinic. Tracking SP moments in a larger sample of culturally diverse, at-risk and, mentally-ill population of mothers and their infants offers the possibility of a simple language- and culture-free measure to identify at-risk dyads. 


\section{Keywords}

Infant; mother-infant interactions; Shared Pleasure moments; maternal mental illness; South Africa

\section{Introduction}

Infant mental health is strongly connected to the quality of caregiving relationships, especially to the mutual adaptation of the infant and caregiver (Mäntymaa et al., 2008). For infants and young children, having a relationship with a responsive, warm, and available caregiver is likely one of the most important factors for positive outcomes (Trevarthen \& Aitken, 2001; Gerhardt, 2004; Feldman, 2007).

The primary goals for the infant during the first postnatal year are the creation of an attachment bond of emotional communication with the primary caregiver and the development of selfregulation (Shore, 2005). The infant, however, has a limited ability to regulate its own emotional as well as physical needs after birth, and a caregiving adult is responsible for what is called mutual regulation (Stern, 1985). When a caregiver is sufficiently sensitive and available emotionally, the infant learns to control their own behaviours and emotions, which in turn may facilitate further social development (Feldman, 2009; Braungart-Rieker, 2001).

Infants develop their ability to experience, express, and regulate positive affective states from the parent-infant interaction, creating the basis for the infants' own emotion regulation (Manian \& Bornstein, 2009; Schore, 2001). Emotion regulation is crucial to mental health and wellness (Cole \& Deater-Deckard, 2009) and may serve as one of the mechanisms through which attachment security affects later socio-emotional outcomes (Braungart, 2001). Empathic care promotes secure attachment in infancy and, by extension, good developmental outcomes in childhood (Bowlby, 1969; Shore, 2001).

An infant's skills for interaction include initiation and maintenance of eye contact, the ability to vocalize and use facial expressions, and head and body movements to engage the caregiver in mutual interaction (Trevarthen \& Aitken, 2001). During episodes of mutual gaze, the mother and infant engage in spontaneous facial, vocal, and gestural communications. Such highly arousing, 
face-to-face interactions allow the infant to be exposed to high levels of social and cognitive information (Shore, 2005). The capacity of the infant to experience increasing levels of positive arousal states is reciprocally regulated by the mother, and depends on her capacity to engage in an interactive communication of emotions (Feldman, 2007). Sharing positive affects in the parentinfant interaction may serve as a foundation for the development of infant self-regulation by fueling early socialization and organization of the infants' experiences (Feldman, 2003; Feldman, 2007). Synchronised shared positive affects in the mother-infant interaction increases the infant's relatedness and, by extension, the development of self-regulation (Feldman et al., 1999; Feldman, 2007). To regulate the high positive arousal, the dyad synchronizes the intensity of their affective behavior within split seconds. These episodes of "affect synchrony" occur in the first expression of social play and generate increasing levels of joy and excitement (Shore, 2005; Feldman et al., 1999).

Paradigms that focus on positive emotions, including joy, happiness and affection (Watson \& Tellegen, 1985), are often considered critical markers of a person's well-being. How a mother's positive emotional state contributes to positive child development in the face of psychosocial adversity or mental illness has garnered greater interest in recent years (Puura et al., 2019; Mäntymaa et al., 2015). From birth, infants are able to engage in face-to-face interactions and, at around 2 months of age, the neutral infant gaze gradually progresses towards positive emotional expressions with gaze contact (Lavelli \& Fogel, 2005). In particular, this face-to-face interaction may elicit a positive effect in both infants and their mothers. For the mother, the infants' smile may serve as a powerful activator of her dopaminergic reward related brain regions (Strathearn, Li, Fonagy \& Montague, 2008).

Puura et al. (2005, 2019) hypothesized that the sharing of a smile or laugh with simultaneous direct gaze contact between a mother and her infant represents a marker of high intensity positive affectivity and named this paradigm "Shared Pleasure (SP)” (Puura et al., 2005, 2019; Mäntymaa et al., 2015). Shared Pleasure moments in parent-infant interaction is defined as "the parent and the child sharing positive affect in synchrony” (Puura et al., 2002). This is expressed in facial expressions such as a laugh, or curving of mouth to smile, together with a direct gaze contact, and a simultaneous or synchronized beginning and ending. The phenomenon of SP was originally 
studied in early mother infant interactions utilizing videos of dyads engaging in free play. Results demonstrated that SP buffered the influence of parental psychopathology on child development and was considered an important feature for fostering positive psychological development (Mäntymaa et al., 2015).

Shared Pleasure is considered a marker of more regulated emotions and serves as a possible screening marker for early identification of at risk dyads (Puura et al., 2002; Mäntymaa et al., 2015, Puura et al., 2019). Most compelling is the potential that a shared positive affect between mother and infant may correlate with attachment security, and may be highly malleable in the first 12 months of life (Mäntymaa et al.,2015).

Maternal mental illnesses reduce the mother's ability to detect and respond to changes in expressions and communication by the infant, which places at risk various aspects of mothering, including attachment and emotion regulation (Walker et al., 2007). Challenges with emotion regulation are the cornerstone of nearly all psychiatric illnesses, and may be associated with behavioural problems in young infants (Suveg, Morelen, Brewer, Thomassin, 2010). Psychiatric illness in the mother or caregiving adult may potentiate these challenges. In a systematic review, Fisher et al. (2012) reported that maternal mental disorders are approximately three times more prevalent in low and middle-income countries (LMICs). In a LMIC like South Africa, mental and reproductive health makes a substantial contribution to the burden of disease in terms of disability, injury and interpersonal violence (Normal et al., 2007). In developing world settings, high rates of maternal mental illnesses, coupled with adverse social conditions, compromise the capacity of caregivers to provide consistent empathic care (Tomlinson, Cooper \& Murray, 2005).

The practice of parenting and the development of attachment patterns naturally vary between the cultural communities that influence child rearing. Learning from developed countries, certain aspects of parenting are consistently related to the cognitive and social-emotional competence in infants (Goodman et al., 2011; Walker et al., 2007). Given the context of ongoing exposure to psychosocial stressors and trauma in their daily lives, practitioners working with parents need an observational measure of parent-infant interaction that would be simple to apply and appropriate in diverse ethnic groups while considering contextual risk exposures. The SP paradigm holds 
promise as a screening observational marker for at risk dyads, with a potential for early identification and intervention. In an ethnically and psychosocially diverse population in South Africa, the need for a reliable but simple observational tool is great. Many of the current tools to measure attachment security and sensitive caregiving have either not been validated in this population, or found to not be culturally sensitive in this diverse population. A paradigm that is not dependent on language or culture, but relies on the observation of natural non-verbal facial expression and gaze contact may be appropriate in this context.

While SP moments as a measurable paradigm has been studied in Europe, a paucity of data exists for developing countries. Given the higher number of adverse life events experienced in developing countries (Fisher et al., 2012) and the higher prevalence of maternal mental health problems in LMICs (Rahman, Surkan, Cayetano, Rwagatare \&, Dickson, 2013), the South African population will provide valuable data in terms of cross-cultural generalizability of SP moments. The ease of applicability and the data generated in this study will help build the foundation for the exploration of early screening tools for infant emotional regulation in a previously unexplored South African population of mothers and their at risk babies. This study, therefore, explored the application of the Shared Pleasure Paradigm on women with and without mental illness who attended a tertiary psychiatric maternal mental health clinic in Cape Town.

\section{Materials and methods}

\section{Study design}

This study forms part of a larger ongoing prospective observational study, The Maternal and Infant Mental Health (MIMH) study, based at Stikland Psychiatric Hospital (Bellville, Western Cape, South Africa). The MIMH study aims to investigate the impact of maternal mental illness and infant measures of social cognitive deficits and oto-acoustic characteristics on mother-child interaction and attachment. The MIMH study recruits participants from a state-based maternal mental health clinic at Stikland Hospital and a private-funded Well Baby follow-up clinic in Bellville, South Africa. The data for this sub-study originated from Stikland Hospital. The main procedures applicable to the current study are briefly outlined below. 


\section{Sample}

We included all mothers with and without psychiatric diagnoses, measured by the MINI interview (Sheehan et al., 1998) and diagnosed according to the Diagnostic and Statistical Manual of Mental Disorders IV criteria (DSM-IV; American Psychiatric Association, 2000), with infants who participated in the MIMH study between 1 April 2013 and 31 December 2016. The sample included 78 dyads who were Caucasian, Mixed-ancestry (Coloured) and Black African (Xhosa), which is in keeping with the population demographics of the study catchment area.

\section{Data collection}

Data was collected when mothers and infants presented at the aforementioned clinics for mental health care follow-up, or for immunisation and physical examination of their baby. As part of the MIMH study, mothers and their infants were scheduled to attend a visit when the baby was between 6 to 12 weeks old and again at 6 months. Demographics and general information about the mothers' health, baby's health, and pregnancy and/or birth pregnancy complications were recorded. Rating scales, including the Maternal-to-Infant Bonding Scale (MIBS) (Taylor et al., 2005), the Maternal Postnatal Attachment Scale (MPAS) (Condon, Corkindale, \& Boyce, 2008),

the Edinburgh Postnatal Depression Scale (EPDS) (Cox, Holden, \& Sagovsky, 1987), and the Recent Life Events Questionnaire (RLEQ) (Brugha, Bebbington, Tennant, \& Hurry, 1985) were recorded individually as part of the parent study.

An interactional video is recorded of the mother communicating with the baby as well as engaging in free play during both visits. Babies range between the ages of 6 weeks to 6 months. For the interactional video, mothers participated in a 10 minute, video-taped free-play session with their infants. Free-play was defined as unrestricted, undirected and unstructured play between mother and infant. The mothers were instructed to play with their infant in the same manner as they usually would. No further instructions were given to the mothers about the use of toys or of how to interact with the infant.

\section{Measuring SP}

We assessed Shared Pleasure moments from a single recorded segment (first 5minutes) of the dyadic free-play video recordings of mothers and infants. The first five minutes are considered 
sufficient for the assessment of positive interactional styles where interrater reliability is good (Kemppinen et al., 2005). SP moments between mother and baby were measured by a single rater (AL) by observing video recordings at full speed. The measurement for Shared Pleasure has three components: the occurrence of a Shared Pleasure moment, the total number of Shared Pleasure moments, and the duration of a Shared Pleasure moment. All possible sequences of SP, from the synchronized dyadic curving of the mouth to a smile together with a direct gaze contact, were tagged if they had a simultaneous beginning and end. The tagged parts of the video recordings were then reviewed at half speed, and the beginnings and endings of SP sequences registered second by second. This process was repeated to ensure that all possible SP moments were tagged. To reduce bias, the rater was blinded to the psychiatric history of the mother.

The first author (AL) received training and achieved reliability in rating SP moments in Finland under the tutelage of the last author (KP). For initial reliability, training videos from a French cohort $(n=11)$ were coded by $\mathrm{AL}$ and reviewed by $\mathrm{KP}$, and then case videos were rated independently by both raters and statistically compared for inter-rater reliability. To assess interrater reliability for this study, 8 videos (10\%) were randomly selected using the random number generator in Excel and were rated by two independent coders (AL and KP). The kappa values for the SP variables used in the analysis, i.e. the occurrence of SP sequences and the mean duration of SP ( $<0.5$ or $>0.5 \mathrm{~s}$ ), were 1.00 and 0.78 , respectively. The respective rates of interrater agreement were $100 \%$ and $87.5 \%$. These rates may be regarded as strong (Healey, 2012; Altman, 1999). Furthermore, none of the SP variables showed a statistically significant difference between the raters, thus showing excellent reliability.

\section{Statistical analysis}

All data were entered in a Microsoft Excel spreadsheet, and analyses were conducted using SAS statistical software (version 9.4, Cary NC). The demographic and clinical characteristics were summarised using frequencies and percentages. From the recorded SP moment information, a binary outcome (yes/no) was coded to indicate whether or not the infant-mother experienced any SP moments, i.e. the occurrence of a SP moment. The next two components of SP moments were recorded: (1) how many SP moments were experienced by the infant-mother pair (frequency), (2) the duration (in sec) of each SP moment. Regression analysis was used to assess the possible 
association between the occurrence of a SP moment and demographic and clinical characteristics of the mothers. A generalized linear mixed model was used with a binomial distribution and a log link was used to model the probability of an occurrence of a SP moment. Univariate unadjusted prevalence (\% and 95\% CIs) and relative risk (PR prevalence ratios) for the various covariates, entered as categorical variables, were reported. The multiple regression model included all the univariate significant risk factors to assess the independent risk factors for SP moments. The statistical significance level for the $\mathrm{F}$ tests was $5 \%$.

\section{Ethical considerations}

The study was approved by the Health Research Ethics Committee (HREC) of Stellenbosch University (Ref \#: S12/04/111A). Study approval was also obtained from the Western Cape Provincial Department of Health for studies conducted at Stikland Hospital. Participation was voluntary, and all mothers provided written informed consent. As the children were under 12 months, consent was gained from the parent. Participation or non-participation in the study had no effect on the standard care offered to mothers and their infants. All data were anonymized using a study number to code for each mother-infant dyad.

\section{Results}

Demographic and clinical characteristics of mothers $(\mathrm{N}=78)$ who participated in this study are summarized in Table 1. The mean maternal age \pm standard deviation (SD) was $30.9 \pm 6.4$ years. Most (72.0\%) mothers were aged between 17 and 34 years and 28.0\% were aged between 35 and 45 years. The mean baby age \pm SD was $66.2 \pm 29.2$ days (median=59.5 days), which is approximately 2 months and 6 days on average. The youngest baby was 27 days old and the oldest was 192 days. Out of a sample of 78 babies, 64 were under 3 months and 14 were over 3 months.

In terms of ethnicity, $51.0 \%$ of the mothers were Caucasian or Mixed-race, while $49.0 \%$ were Black/African. The majority of the sample was unemployed (94.0\%), and most of the participants (82.0\%) earned less than R10 000 (700 USD) per month. More than half the participants (55.0\%) were either divorced, single or separated from their life partner, while $45 \%$ were married. Half (54.0\%) of the participants had a diagnosed psychiatric illness, the most common being psychosis 
or schizophrenia spectrum disorder (33.3\%), major depressive disorder (33.3\%), and bipolar disorder (21.4\%).

For the 78 dyad pairs assessed, SP moments occurred in only 20.5\% $(n=16)$. Further analysis of SP moments (Table 2) demonstrated that a single SP moment lasted as least 0.5 seconds in 24.0\% of the SP positive sample, with the longest duration of an SP moment lasting 2 seconds (27.0\%). The majority of SP moments (46.0\%) lasted at least 1 second.

Of the 64 babies (that were younger than 3 months), 22.0\% ( $n=14)$ and 14.0\% $(n=2)$ of the 64 babies were older than 3 months had an SP moment. The length of the video sequences did not appear to account for the absence of SP moments. SP moments were not observed even when extended (greater than 5 minutes in duration) video sequences were evaluated.

For mothers who experienced SP moments, 21.4\% $(n=12)$ were between the ages of 17 and 34 years, while $18.2 \%(n=4)$ were older than 35 years. The numbers for the individual ethnic groups were Caucasian n=6, Mixed-race n=34, Blacks n=36 and African n=2. (Table 3). Similar ethnic groups were combined for analysis due to small numbers. A SP moment was significantly more likely to occur in Black/African mothers (31.6\%; p=0.033) compared to Mixed/Caucasian race mothers (10\%), as well as in mothers who were married (34.3\%; $\mathrm{p}=0.016)$ compared to unmarried mothers (9.3\%) (Table 3). The chance of a SP moment was 3 times more likely in the Black/African group compared to the Mixed-race/Caucasian group. Mothers without mental illness also had significantly more SP moments (33.3\%, p= 0.021) than mothers with mental illness (9.5\%). More mothers that did not have SP moments acknowledged current use of substances (22.0\%) compared to mothers with SP moments (17.9\%) but this difference was not significant $(\mathrm{p}=0.668)$.

The multiple regression model included the significant covariates from the univariate model, race, marital status and psychiatric illness. The results show that not being married was the only variable that was significantly associated with the occurrence of SP moments (34\% of married and 9\% of unmarried women having SP moments; $\mathrm{p}=0.027$ ). Married women are 3.7 times more likely to have an SP moment with their babies compared to unmarried women. The multiple regression 
model is probably underpowered to detect other significant associations due to the small sample size.

\section{Discussion}

In infant research, direct observation of behaviours are thought to provide more sensitive and accurate data than questionnaires and interviews (Aspland \& Gardner, 2003). Most observation methods, however, were developed in Western developed countries with little room for application in ethnically diverse populations. South African mothers and their experiences cannot be homogenized or generalized if we recognize the historical, social and political influences on racial and linguistic groups. In this study, the Shared Pleasure paradigm was applied to video recorded observation of mothers and their infants, and was measured according to the strict SP Paradigm descriptions (Puura et al., 2002). This is the first application of the SP paradigm in an ethnically diverse sample outside of a Eurocentric clinical setting.

Puura et al. (2002) demonstrated that mothers and infants with good interactional skills had more moments of Shared Pleasure. In a Finnish sample of 2 month old infants and their mothers, SP moments occurred in $80.0 \%$ of dyads (Latva, Mäntymaa, Puura, Luoma, Salmelin, Tamminen, 2013) with the median SP sequence length of 1.0 second. In older infants (7 months old) in the same population, the occurrence of SP was 67.0\%. The number and proportion of SP moments in this Finnish normal population were positively correlated with higher infant involvement with the parent, infant responsiveness and greater maternal sensitivity as measured by the Emotional Availability Scale (Biringen, Robinson \& Emde, 2000).

Contrary to expectations, very few SP moments (20.5\%) were observed in the South African sample, even within interactions between infants and their mentally-well mothers. More than two decades following democracy, ordinary South Africans continue to be at risk of multiple socioeconomic adversities such as poverty, intimate partner violence, mental illness, substance use and malnutrition (Tomlinson et al., 2014) with infants from poorer communities being at risk of life long socio-emotional and developmental vulnerability. Infants in this situation may be especially vulnerable to harsh or distant parenting under the strain of marked socio-economic hardship (compounded by exposure to violence and substance misuse) that has been linked with 
lower maternal sensitivity and lower infant engagement with mothers (Cooper et al., 2002). We speculate that this may in part explain the unexpectedly low occurrence of SP moments in the general sample.

In those dyads who did share SP moments, $46.0 \%$ of the SP sequences lasted at least 1 second, similar to international studies (Latva et al, 2013; Mäntymaa et al., 2015). The presence of more SP moments in infants under the age of 3 months is also in keeping with the Finnish experience of more SP moments in younger infants (Latva et al., 2013). This coincides developmentally with the first bio-behavioural shift between ages 2 to 3 months that is characterized by the onset the social smile.

It is suggested that parental mental illness may profoundly affect the parent's ability to reciprocate emotion and regulate their infants (Schore, 2001; Suveg, Shaffer, Morelen \& Thomassin, 2011). Our sample did demonstrate low initial frequencies (20.5\%) of SP moments in the total sample. However, when the sample is stratified for psychiatric illness, mothers without mental illnesses experienced significantly more SP moments (33.3\%) than mothers with mental illness (9.5\%; $\mathrm{p}=$ 0.021). Maternal mental illness reduces the mother's ability to detect and respond to changes in expressions and communication by the infant (Feldman, Magori-Cohen, Galili, Singer, Louzoun, 2011). The current study supports research conducted by Mäntymaa et al., (2015) who hypothesized that the presence of maternal mental illness would limit the capacity for the mother and infant to experience Shared Pleasure moments.

Significantly more married women experienced an SP moment with their babies than single/unmarried women $(\mathrm{p}=0.027)$, with the regression model showing that married women are 3.7 times more likely to have an SP moment. There are many theories to suggest that women who are married are likely to be more emotionally and socio-economically supported than their unmarried counterparts. A review by Hetherington \& Stanley-Hagan (1999) reported that the consequence of single parenthood (particularly following the separation of parents) is often financial hardship and lower socio-economic status. This, in turn, exacerbates mother's emotional distress that impairs or has an adverse effect on parenting for single mothers. The underlying predictor of positive maternal attitudes, with regard to interactive behaviours in infants under the 
age of 4 months, was found to be significantly associated with intimate support and quality of marital relationships (Crnic, Greenberg, Ragozin, Robinson \& Basham, 1983). A Canadian study (Muhammad \& Gagnon, 2010) reported that motherhood did not affect perceived stress among married or cohabitating women. However, this previous study also demonstrated that single and divorced mothers endured the highest levels of stress and this, in turn, appeared to affect perceived parental efficacy. These studies, albeit international, suggest that maternal perceived efficacy and stress levels influence maternal capacities to sensitively and positively interact with babies, and this could support the finding that SP moments were more commonly experienced amongst the married participants in our local study.

Education and financial status and, by inference, socioeconomic status may influence parenting (Tomlinson, Cooper \& Murray, 2005; Cooper et al., 2002), and this additional stress influences the mother's ability to respond sensitively to and interpret interactional cues. However, in the current study, there was no statistically significant relationship between education level or financial status and SP moments.

Black/African mothers in our sample experienced significantly more SP moments with their infants compared with mothers of other race groups. Culture influences a mothers' behavior toward her infant, and different ethnic groups may emphasize different dimensions of interactions with their children. It is important to consider South Africa's history when considering how cultural influences impact on motherhood in different communities, and that it may be problematic to extrapolate that all mothers belonging to a particular cultural community have the same experience, especially in contemporary South Africa. Research that addresses the experiences of mothers and their interactions with their babies living in the South African context is limited. Magwaza (2003) suggested that the social and cultural contexts in which Black mothers live shape their perspectives and behaviours related to sensitive mothering. In a doctoral thesis by Dale (2013) that explored the narrative understanding of urban Black South African mothers, participants spoke about enacting "good mothering" through meeting the needs of their babies by providing for them "emotionally" rather than physically. The sentiment was to offer their babies a good example of mothering, despite the adverse environmental and social challenges, and this translated into providing babies with optimal mothering experiences. One could speculate that the Black mothers 
in our sample may also have had similar intentions and that providing joyous and pleasurable experiences between themselves and their infants may be seen as an example of providing positive role modelling. However, we acknowledge that the experience of motherhood is multifaceted and complex, and this sample is too limited to make further inferences. Ethnic and cultural differences within this sample may explain the difference between race and SP moments, and future studies involving a larger cohort with further explore this hypothesis.

Limitations of this study include the small sample size of mother baby pairs. In addition, the small number of observed SP moments limited the statistical analyses that could be formed in this preliminary investigation. Furthermore, the small sample size means the multiple regression model may be underpowered to detect significant associations.

\section{Conclusions}

This study used a language- and culture-free paradigm "the Shared Pleasure moment" of synchronized direct eye gaze contact and simultaneous smile between mothers and their infants to assess reciprocal positive interaction in a group of mothers attending a maternal mental health clinic in Cape Town. While results demonstrated a low overall occurrence of SP moments in both groups of mothers (that is, mothers with and without mental illnesses), there were significant associations between SP and race group, marital status and the presence of mental illness. Tracking SP moments in a broader sample of a culturally diverse, at-risk and mentally-ill population of mothers and their infants could be simple and easily identifiable observable measure to identify at-risk dyads. The benefit of this measure is that it does not rely on education or a sophisticated understanding of infant responses on the part of the mother. The role of the infant's positive affectivity could influence the capacity for SP in its interaction with the mother (Varpula, 2014); however, as our study did not assess the infant's withdrawal or inhibition at baseline, correlations between the presence of Shared Pleasure and the level of positive affectivity in the infant was not determined.

The findings in this study were preliminary and would need to be further confirmed and correlated with sociodemographic variables. This study will serve as the foundation for a more comprehensive exploration of SP moments as a screening tool in a local population. The follow- 
up study will assess correlations with infant developmental outcomes and more robust measures of maternal sensitivity and reciprocity.

\section{Acknowledgements}

We acknowledge Dr Karis Moxley (Department of Psychiatry, Stellenbosch University) for critical feedback, writing assistance and technical editing.

\section{Declaration of interest}

No potential conflict of interest was reported by the authors.

\section{References}

Altman, D.G. (1999). Practical statistics for medical research. Chapman \& Hall/CRC, London.

American Psychiatric Association. (2000). Diagnostic and Statistical Manual of Mental Disorders (Revised 4th ed.). Washington, DC.

Aspland, H., \& Gardner, F. (2003). Observational measures of parent-child interaction: An introductory review. Child and Adolescent Mental Health, 8(3), 136-143.

Biringen, Z., Robinson, J. L., \& Emde, R. N. (2000). Appendix B: The emotional availability scales (an abridged infancy/early childhood version). Attachment \& human development, 2(2), 256-270.

Bowlby, J. (1969). Attachment and loss v. 3 (Vol. 1). Random House. Furman, W., \& Buhrmester, D. (2009). Methods and measures: The network of relationships inventory: Behavioral systems version. International Journal of Behavioral Development, 33, 470-478

Braungart-Rieker, J. M., Garwood, M. M., Powers, B. P., \& Wang, X. (2001). Parental sensitivity, infant affect, and affect regulation: Predictors of later attachment. Child development, 72(1), 252-270.

Brugha, T., Bebbington, P., Tennant, C., \& Hurry, J. (1985). The list of threatening experiences: a subset of 12 life event categories with considerable long-term contextual threat. Psychological medicine, 15(1), 189-194.

Cole, P. M., \& Deater-Deckard, K. (2009). Emotion regulation, risk, and psychopathology. Journal of Child Psychology and Psychiatry. 
Cooper, P. J., Landman, M., Tomlinson, M., Molteno, C., Swartz, L., \& Murray, L. (2002). Impact of a mother-infant intervention in an indigent peri-urban South African context: pilot study. The British Journal of Psychiatry, 180(1), 76-81.

Condon, J. T., Corkindale, C. J., \& Boyce, P. (2008). Assessment of postnatal paternal-infant attachment: development of a questionnaire instrument. Journal of Reproductive and Infant Psychology, 26(3), 195-210.

Cox, J. L., Holden, J. M., \& Sagovsky, R. (1987). Detection of postnatal depression: development of the 10-item Edinburgh Postnatal Depression Scale. The British Journal of Psychiatry, 150(6), 782-786.

Crnic, K. A., Greenberg, M. T., Ragozin, A. S., Robinson, N. M., \& Basham, R. B. (1983). Effects of stress and social support on mothers and premature and full-term infants. Child development, 209-217

Dale, L. K. (2013). A narrative understanding of the maternal experience of urban black South African mothers (Doctoral dissertation).

Fisher, J., Mello, M. C. D., Patel, V., Rahman, A., Tran, T., Holton, S., \& Holmes, W. (2012). Prevalence and determinants of common perinatal mental disorders in women in low-and lower-middle-income countries: a systematic review. Bulletin of the World Health Organization, 90, 139-149.

Feldman, R. (2007). Parent-infant synchrony and the construction of shared timing; physiological precursors, developmental outcomes, and risk conditions. Journal of Child psychology and Psychiatry, 48(3-4), 329-354.

Feldman, R. (2003). Infant-mother and infant-father synchrony: The coregulation of positive arousal. Infant Mental Health Journal: Official Publication of The World Association for Infant Mental Health, 24(1), 1-23.

Feldman, R., Granat, A., Pariente, C., Kanety, H., Kuint, J., \& Gilboa-Schechtman, E. (2009). Maternal depression and anxiety across the postpartum year and infant social engagement, fear regulation, and stress reactivity. Journal of the American Academy of Child \& Adolescent Psychiatry, 48(9), 919-927.

Feldman, R., Greenbaum, C. W., \& Yirmiya, N. (1999). Mother-infant affect synchrony as an antecedent of the emergence of self-control. Developmental psychology, 35(1), 223. 
Feldman, R., Magori-Cohen, R., Galili, G., Singer, M., \& Louzoun, Y. (2011). Mother and infant coordinate heart rhythms through episodes of interaction synchrony. Infant Behavior and Development, 34(4), 569-577.

Gerhardt, S. (2004). Why love matters: How affection shapes a baby's brain. Routledge.

Goodman, S. H., Rouse, M. H., Connell, A. M., Broth, M. R., Hall, C. M., \& Heyward, D. (2011). Maternal depression and child psychopathology: a meta-analytic review. Clinical child and family psychology review, 14(1), 1-27.

Healey, J. F. (2012). Statistics: A tool for social research (9th ed.). Belmont, CA: Wadsworth Cengage Learning.

Hetherington, E. M., \& Stanley-Hagan, M. (1999). The adjustment of children with divorced parents: A risk and resiliency perspective. The Journal of Child Psychology and Psychiatry and Allied Disciplines, 40(1), 129-140.

Kemppinen, K., Kumpulainen, K., Räsänen, E., Moilanen, I., Ebeling, H., Hiltunen, P., \& Kunelius, A. (2005). Mother-child interaction on video compared with infant observation: Is five minutes enough time for assessment?. Infant Mental Health Journal: Official Publication of The World Association for Infant Mental Health, 26(1), 69-81.

Lavelli, M., \& Fogel, A. (2005). Developmental changes in the relationship between the infant's attention and emotion during early face-to-face communication: the 2-month transition. Developmental psychology, 41(1), 265.

Latva, R., Mäntymaa, M., Puura, K., Luoma, I., Salmelin, R.K., Tamminen, T. (2013). Shared Pleasure in early infancy: building resilience? Poster presentation

Magwaza, T. (2003). Perceptions and experiences of motherhood: A study of black and white mothers of Durban, South Africa. Jenda: A Journal of Culture and African Women Studies, (4).

Manian, N., \& Bornstein, M. H. (2009). Dynamics of emotion regulation in infants of clinically depressed and nondepressed mothers. Journal of Child Psychology and Psychiatry, 50(11), 1410-1418.

Mäntymaa, M., Puura, K., Luoma, I., Kaukonen, P., Salmelin, R. K., \& Tamminen, T. (2008). Infants' social withdrawal and parents' mental health. Infant Behavior and Development, 31(4), 606-613. 
Mäntymaa, M., Puura, K., Luoma, I., Latva, R., Salmelin, R. K., \& Tamminen, T. (2015). Shared Pleasure in early mother-infant interaction: predicting lower levels of emotional and behavioral problems in the child and protecting against the influence of parental psychopathology. Infant mental health journal, 36(2), 223-237

Muhammad, A., \& Gagnon, A. (2010). Why should men and women marry and have children? Parenthood, marital status and self-perceived stress among Canadians. Journal of health psychology, 15(3), 315-325.

Norman, R., Bradshaw, D., Schneider, M., Joubert, J., Groenewald, P., Lewin, S., ... \& Nojilana, B. (2007). A comparative risk assessment for South Africa in 2000: towards promoting health and preventing disease. South African Medical Journal, 97(8), 637-641.

Puura, K., Davis, H., Mäntymaa, M., Tamminen, T., Roberts, R., Dragonas, T., ... \& Leontiou, F. (2005). The outcome of the European Early Promotion Project: mother-child interaction. International Journal of Mental Health Promotion, 7(1), 82-94.

Puura, K., Leppänen, J., Salmelin, R., Mäntymaa, M., Luoma, I., Latva, R., Peltola, M., Lehtimäki, T., Tamminen, T.(2019). Maternal and infant characteristics connected to Shared Pleasure in dyadic interaction. Infant Mental Health Journal (in press)

Puura, K., Davis, H., Papadopoulou, K., Tsiantis, J., Ispanovic-Radojkovic, V., Rudic, N., ... \& Vizakou, S. (2002). The European Early Promotion Project: A new primary health care service to promote children's mental health. Infant Mental Health Journal: Official Publication of The World Association for Infant Mental Health, 23(6), 606-624.

Rahman, A., Surkan, P. J., Cayetano, C. E., Rwagatare, P., \& Dickson, K. E. (2013). Grand challenges: integrating maternal mental health into maternal and child health programmes. PLoS Medicine, 10(5), e1001442.

Schore, A. N. (2001). The effects of early relational trauma on right brain development, affect regulation, and infant mental health. Infant mental health journal, 22(1-2), 201-269.

Schore, A. N. (2005). Attachment, affect regulation, and the developing right brain: Linking developmental neuroscience to pediatrics. Pediatrics in review, 26(6), 204-217. 
Sheehan, D. V., Lecrubier, Y., Sheehan, K. H., Amorim, P., Janavs, J., Weiller, E., ... \& Dunbar, G. C. (1998). The Mini-International Neuropsychiatric Interview (MINI): the development and validation of a structured diagnostic psychiatric interview for DSM-IV and ICD-10. The Journal of clinical psychiatry.

Stern, D. N. (1985). The interpersonal world of the infant: A view from psychoanalysis and developmental psychology. Karnac Books.

Strathearn, L., Li, J., Fonagy, P., \& Montague, P. R. (2008). What's in a smile? Maternal brain responses to infant facial cues. Pediatrics, 122(1), 40-51.

Suveg, C., Morelen, D., Brewer, G. A., \& Thomassin, K. (2010). The emotion dysregulation model of anxiety: A preliminary path analytic examination. Journal of Anxiety Disorders, 24(8), 924-930

Suveg, C., Shaffer, A., Morelen, D., \& Thomassin, K. (2011). Links between maternal and child psychopathology symptoms: Mediation through child emotion regulation and moderation through maternal behavior. Child Psychiatry \& Human Development, 42(5), 507.

Taylor, A., Atkins, R., Kumar, R., Adams, D., \& Glover, V. (2005). A new Mother-to-Infant Bonding Scale: links with early maternal mood. Archives of Women's Mental Health, 8(1), 4551.

Tomlinson, M., Cooper, P., \& Murray, L. (2005). The mother-infant relationship and infant attachment in a South African peri-urban settlement. Child development, 76(5), 1044-1054.

Tomlinson, M., O’Connor, M. J., Le Roux, I. M., Stewart, J., Mbewu, N., Harwood, J., \& Rotheram-Borus, M. J. (2014). Multiple risk factors during pregnancy in South Africa: the need for a horizontal approach to perinatal care. Prevention Science, 15(3), 277-282.

Trevarthen, C., \& Aitken, K. J. (2001). Infant intersubjectivity: Research, theory, and clinical applications. The Journal of Child Psychology and Psychiatry and Allied Disciplines, 42(1), 3-48.

Varpula, R. (2014). Maternal mood, infant temperament and shared joy in mother-child interaction (Master's thesis). 
Walker, S. P., Wachs, T. D., Gardner, J. M., Lozoff, B., Wasserman, G. A., Pollitt, E., ... \& International Child Development Steering Group. (2007). Child development: risk factors for adverse outcomes in developing countries. The lancet, 369(9556), 145-157.

Watson, D., \& Tellegen, A. (1985). Toward a consensual structure of mood. Psychological bulletin, 98(2), 219.

\section{Tables}

Table 1: Demographic and clinical characteristics of mothers at their first visit $(\mathrm{n}=78)$

\begin{tabular}{lc}
\hline Variable & Frequency, n (\%) \\
\hline Age & \\
17-34 years & $56(72)$ \\
35-45 years & $22(28)$ \\
Race & \\
Caucasian/Mixed race & $40(51)$ \\
Black/African & $38(49)$ \\
Family Income & \\
<R10 000p.m. & $64(82)$ \\
>=R10 000p.m. & $14(18)$ \\
Employment Status & \\
Employed & $5(6)$ \\
Unemployed/on maternity leave & $73(94)$ \\
Marital Status & \\
Married & $35(45)$ \\
Separated, Divorced, Single, Other & $43(55)$ \\
Mental Health Status & \\
With Mental illness & \\
Without Mental illness & $42(54)$ \\
\hline
\end{tabular}


Table 2: Frequency and duration of SP moments

\begin{tabular}{lcccccccc}
\hline Duration of SP & \multicolumn{7}{c}{ Frequency, n (\%) } \\
\cline { 2 - 8 } moment & SP & SP & SP & SP & SP & SP & SP & SP \\
& Moment & Moment & Moment & Moment & Moment & Moment & Moment & Moment \\
& 1 & 2 & 3 & 4 & 5 & 6 & 7 & 1 to 7 \\
\hline 0.5 seconds & $3(19)$ & $3(33)$ & $1(17)$ & $0(0)$ & $1(50)$ & $0(0)$ & $1(100)$ & $9(24)$ \\
1 second & $8(50)$ & $4(44)$ & $3(50)$ & $2(100)$ & $0(0)$ & $0(0)$ & $0(0)$ & $17(46)$ \\
2 seconds & $5(31)$ & $1(11)$ & $2(33)$ & $0(0)$ & $1(50)$ & $1(100)$ & $0(0)$ & $10(27)$ \\
3 seconds & $0(0)$ & $0(0)$ & $0(0)$ & $0(0)$ & $0(0)$ & $0(0)$ & $0(0)$ & $0(0)$ \\
4 seconds & $0(0)$ & $1(11)$ & $0(0)$ & $0(0)$ & $0(0)$ & $0(0)$ & $0(0)$ & $1(3)$ \\
Total & $16(100)$ & $9(100)$ & $6(100)$ & $2(100 \%)$ & $2(100 \%)$ & $1(100)$ & $1(100)$ & $37(100)$ \\
\hline
\end{tabular}


Table 3: The number (n and \%) of women with an occurrence of a SP moment for the various categories of the demographic and clinical characteristics at the $1^{\text {st }}$ visit $(\mathrm{n}=78)^{* *}$

\begin{tabular}{|c|c|c|c|c|}
\hline Variable & Total $(\mathrm{N}=78)$ & $\mathbf{n}$ & $\%$ & p-value \\
\hline \multicolumn{5}{|l|}{ Age } \\
\hline 17-34 years & 56 & 12 & 21.4 & \\
\hline $35-45$ years & 22 & 4 & 18.2 & 0.753 \\
\hline $\begin{array}{l}\text { Race } \\
\text { Caucasian n=6 /Mixed race } \\
\text { n=34 }\end{array}$ & 40 & 4 & 10 & \\
\hline Black $n=36$ /African $n=2$ & 38 & 12 & 31.6 & $* 0.033$ \\
\hline \multicolumn{5}{|l|}{ Family Income } \\
\hline$<\mathrm{R} 10000$ & 64 & 14 & 21.9 & \\
\hline$>$ R10000 & 14 & 2 & 14.3 & 0.542 \\
\hline $\begin{array}{l}\text { Marital Status } \\
\text { Married }\end{array}$ & 35 & 12 & 34.3 & \\
\hline $\begin{array}{l}\text { Separated, Divorced, Single, } \\
\text { Other }\end{array}$ & 43 & 4 & 9.3 & $* 0.016$ \\
\hline \multicolumn{5}{|l|}{ Education Level $^{\ddagger}$} \\
\hline Primary (Grades 1 to 7 ) & 6 & 0 & 0 & - \\
\hline Secondary (Grades 8 to 12 ) & 64 & 15 & 23.4 & \\
\hline Other(technical or tertiary) & 8 & 1 & 12.5 & \\
\hline $\begin{array}{l}\text { Employment Status } \\
\text { Employed }\end{array}$ & 5 & 1 & 20 & \\
\hline $\begin{array}{l}\text { Unemployed/on maternity } \\
\text { leave } \\
\text { Current Maternal Substance } \\
\text { Abuse }\end{array}$ & 73 & 15 & 20.6 & 0.977 \\
\hline Yes & 28 & 5 & 17.9 & \\
\hline $\begin{array}{l}\text { No } \\
\text { Mental Health Status }\end{array}$ & 50 & 11 & 22 & 0.668 \\
\hline With Mental illness & 42 & 4 & 9.5 & \\
\hline Without Mental illness & 36 & 12 & 33.3 & $* 0.021$ \\
\hline
\end{tabular}

${ }^{\ddagger}$ Sample size does not meet assumptions for statistical analyses

The \% in Table 3 indicate the number and \% of mothers with a SP moment (occurrence) and the $p$ value indicate if the occurrence is different for the 2 ethnic groups.

*Significant at a p-value of $<0.05$

**a positive response of experiencing a SP moment was modeled as the outcome 